\title{
Wahrheit und Modalität
}

\author{
VOLKMAR ENGERER \\ Statsbiblioteket, Århus, Danmark
}

\begin{abstract}
Dette bidrag er en systematisk gennemgang af aktuelle tilgange til modalitet, som fremhæver modalitetens fællestræk på trods af de forskellige vinkler, som findes i modalitetsforskning. Sandhed, eller bedre sagt: sandhed med modifikationer, er nøglen til forståelsen af denne universale kategori. Problematisk-problematiseret sandhedsreference som kernen $\mathrm{i}$ modalitet har to korrelater i den aktuelle forskning, den logisk-semantiske og den pragmatiske gren, som ikke er modstandere, men - tværtimod - er to nødvendige vinkler på et og samme fænomen. I første del af denne artikel belyses typisk logisk-semantiske studier, som åbner vejen fra uanalyserede modaliserede propositioner hen til umodaliserede, men verificerbare propositioner, hvor det resulterende kompleks, bestående af proposition plus formale repræsentanter af modale betydninger, kan underkastes en modelteoretisk interpretation. Den anden og sidste del af det foreliggende arbejde undersøger den pragmatiske gren inden for modalitet, hvor talerens forpligtelse til sprogligt at tage stilling til sit problematiske forhold til sandhedsværdien i sin ytring kommer i spil.
\end{abstract}

\section{EINLEITUNG - LOGISCH-SEMANTISCHE UND PRAGMATISCHE MODALITÄT}

Modalität ist die semantisch-pragmatische Kategorie, welche es auf systematische Weise erlaubt, die Standardeigenschaft von Propositionen, sich auf Sachverhalte in der aktuellen und wirklichen Welt $\mathrm{zu}$ beziehen, auf Sachverhalte in anderen Welten zu erweitern, die mit „unserer“ nicht identisch sind. So oder ähnlich lautete der erste Satz in meiner Themabeschreibung zum 5. Forschungskolloquium an der Staatsbibliothek 2004, das unter dem Motto 
"Modalität" stand. Ich denke, der Grundgedanke ist immer noch richtig: Das modale Inventarium ${ }^{1}$ natürlicher Sprachen (modale Adverbien, Verben, syntaktische Konstruktionen usw., s. Bußmann 2002: 438f.) gibt dem Sprecher ungeheuer machtvolle Mittel in die Hand, seine Diskurswelt auf z.B. nichtexistierende, aber mögliche oder gewünschte, sogar unmögliche Dinge und Sachverhalte auszudehnen ${ }^{2}$.

Mit dem Sprechen über Mögliches und andere für den Realisten wenig greifbare Dinge ist den Diskursteilnehmern die wichtige Option erschwert, den Wahrheitswert geäußerter Propositionen, z.B. von Konstativen, in der aktuellen Äußerungssituation oder sogar in der wirklichen Welt zu verifizieren. Sind also modale Ausdrücke lügnerisch? Natürlich nicht, aber Aufrichtigkeit und Wahrheitstreue ist ihnen sozusagen mitgegeben, als Sicherheitsveranstaltung, die das empiriefeindliche Potential der Modalität in Schach halten soll. Der modalitätstypische Wahrheitsbezug, der mich hier beschäftigen wird, hat zwei Aspekte, welche zwei sprachlichen bzw. linguistischen Dimensionen entsprechen. Da ist einmal die logische Basis der Bedeutungskonstruktion modalitätshaltiger Ausdrücke; sie ist das Fundament, das den Weg zur Bedeutung, zu verifizierbaren, modalitätslosen Propositionen - und damit zur Wahrheit oder Falschheit von bedeutungstragenden modalen Sätzen vorzeichnet. Es ist der logisch-semantische Aspekt von Modalität, der modale Ausdrücke evaluierbar macht und sie damit der Wahrheit verpflichtet ${ }^{3}$. Der andere Aspekt ist in der wirklichen Kommunikation verhaftet, die niemals wertfrei geschieht; Wahrheit oder Wahrhaftigkeit gehört zu den grundlegendsten Werten kommunikativen Verhaltens bzw. kommunikativer Kooperation. Da Modalität, wie oben schon angedeutet, in sich den Keim des (Un-)Wahrscheinlichen trägt, ist der Äußerer in die Pflicht gerufen, sein problematisches Verhältnis zum Wahrheitsgehalt der geäußerten Proposition in irgendeiner Weise klarzustellen (unterlässt er dies und kann er dieses Wissen auch dem Partner nicht unterstellen, kommt es womöglich zu einer Lüge). Die Modalisierung von Ausdrücken ist das herausragende sprachliche Mittel, das dem Sprecher erlaubt, aus der pauschalen Verantwortung für kategoriale Wahrheitswerte zeitweise auszubrechen. Dieser Aspekt von Modalität ist pragmatisch, weil er das Verhältnis des Sprechers sowohl zum denotativen Gehalt der Äußerung als auch zum Hörer beinhaltet. 
Beide Aspekte von Modalität, der logisch-semantische und der pragmatische, gründen daher in einem gemeinsamen Wahrheitsbegriff, was Modalität als einheitliche semantisch-pragmatische Kategorie, wie einleitend formuliert, konstituiert. Diese Verankerung in beiden Dimensionen ist allerdings bisher nicht genügend erkannt worden, ebenso wenig wie eine problematische (problematisierte) Wahrheit als Kern der Modalitätskategorie in den Vordergrund gestellt worden ist. Darstellungen von Modalität fokussieren oft entweder auf den logisch-semantischen oder den pragmatischen Aspekt - oder versuchen, den Wahrheitsbegriff in der Analyse zu umgehen. Beides wird Modalität nicht gerecht, und eine integrierte Analyse dieser Kategorie steht meiner Meinung nach noch aus. Ich werde dies im Folgenden auch nicht leisten können. Was ich mir vorgenommen habe, ist, einige Standarddarstellungen von Modalität vorzustellen und sie auf die beiden Dimensionen (logisch-semantisch - pragmatisch) und den Wahrheitsbegriff hin zu untersuchen. Ich hoffe, dies wird meine programmatischen Behauptungen etwas plausibler machen.

\section{LOGISCH-SEMANTISCHE ASPEKTE VON MODALITÄT}

\subsection{Die Standardanalyse modaler Ausdrücke}

In der Modalitätsforschung werden, wie allgemein bekannt ist, drei Typen von Modalität unterschieden, alethische, epistemische ${ }^{4}$ und deontische Modalität (vgl. z.B. Lyons 1977: 791). Allen drei liegen die Begriffe der Notwendigkeit und Möglichkeit zugrunde (Lyons 1977: 787), am deutlichsten wohl der alethischen Modalität, die sozusagen die Ausgangsanalyse für die beiden anderen anbietet. Der Tradition zufolge werden hier zunächst (amodale) Propositionen in kontingent sowie nicht-kontingent wahre und falsche Propositionen eingeteilt (Lyons 1977: 787, Cann 1993: 270f.), ${ }^{5}$ was zu einer viergliedrigen Typologie führt:

\begin{tabular}{|l|l|l|}
\hline & wahr & falsch \\
\hline kontingent & I & II \\
\hline nicht-kontingent & III & IV \\
\hline
\end{tabular}

Diese Typologie von Propositionen kann ausgenutzt werden, um den ersten Schritt hin zu einer Interpretation modaler Ausdrücke auf den beiden 
Dimensionen notwendig/möglich $\mathrm{zu}$ tun. Notwendig sowie möglicherweise wahre und falsche Propositionen, also kunstsprachliche Übersetzungen modaler Ausdrücke in einer natürlichen Sprache, werden im Rahmen der alethischen Modallogik eindeutig auf die vier obigen, nicht-modalen propositionalen Typen abgebildet. In Anlehnung an (Cann 1993: 271f.) besteht folgende Zuordnung:

$\begin{array}{ll}\text { Notwendig wahr: }^{6} & \text { III } \\ \text { Notwendig falsch: } & \text { IV } \\ \text { Möglicherweise wahr: } & \text { I + II + III } \\ \text { Möglicherweise falsch: } & \text { I + II + IV }\end{array}$

Damit ist die Möglichkeit gegeben, die Interpretation modaler Sätze auf die Interpretation unmodalisierter Propositionen zurückzuführen. Sätze wie (Beispiele aus Cann: 1993: 270)

(1) Every proposition is necessarily either true or false, but not both

(2) Every proposition must be either true or false, but not both

(3) Bertie possibly knows that the Morning Star is the Evening Star

(4) Bertie may know the Morning Star is the Evening Star

sind wahr genau dann, wenn der unmodalisierte Rest (every proposition is either true or false, but not both bzw. Bertie knows that the Morning Star is the Evening Star) zum Typ III (notwendig wahr) bzw. zum Typ I, II oder III (möglicherweise wahr) gehört. In der formalen Semantik repräsentiert man daher Notwendigkeit und Möglichkeit durch Operatoren ( $\square$ bzw. $\diamond$ ), die auf modallosen Ausdrücken (hier: Formeln) operieren. Die modallosen Formeln werden dann in einem Modell $M$ anhand einer Wertezuweisungsfunktion für Variablen $g$ sowie einer möglichen Welt $w_{n}$ interpretiert (Cann 1993: 272; zur Möglichen-Welt-Semantik vgl. auch Chierchia/McConnell-Ginet 1990: 208ff.). Die Wahrheitsbedingungen für die beiden logischen Modaloperatoren $\square$ und $\diamond$ sehen dann wie folgt aus (vgl. Cann 1993: 273f.):

(5) Wenn $\Phi$ eine Formel ist, dann ist $[\square \Phi]^{\mathrm{M}, \mathrm{gwn}}$ wahr gdw. $[\Phi]^{\mathrm{M}, \mathrm{gwm}}$ wahr ist für alle $\mathrm{w}_{\mathrm{m}}$ in $\mathrm{W}$

(6) Wenn $\Phi$ eine Formel ist, dann ist $[\diamond \Phi]^{\mathrm{M}, \mathrm{gwn}}$ wahr gdw. $[\Phi]^{\mathrm{Mgg,wm}}$ wahr ist für ein $\mathrm{w}_{\mathrm{m}}$ in $\mathrm{W}$ 
( $W$ ist eine Menge möglicher Welten.) Alethische Modalität begründet also die Wahrheitsbedingungen von Sätzen, die in Formeln einer logischen Kunstsprache mit dem Notwendigkeitsoperator $\square$ oder dem Möglichkeitsoperator $\diamond$ übersetzt werden können. Die Wahrheitsbedingungen solcher Formeln werden dadurch ermittelt, dass zunächst die Operatoren von der Formel entfernt werden und die Restformel $(\Phi)$ in den möglichen Welten des Modells evaluiert wird. Im Falle von $\square$ müssen alle Welten $w_{\mathrm{m}}$ durchgegangen werden (Cann 1993: 273), im Falle von $\diamond$ genügt eine Welt, in der $\Phi$ wahr ist. Der Grundgedanke dieses Vorgehens kann mit folgendem Zitat noch einmal deutlich gemacht werden:

„Things might be, or might have been, other than they actually are, or were. To conceive of a state of affairs being otherwise is to conceive of is being true or real in some nonactual world(s) [...]. The essence of ,modality' consists in the relativization of the validity of sentence meanings to a set of possible worlds." (Kiefer 1994: 2515)

Die alethischen Modallogik, ${ }^{7}$ welche die logisch-semantische Komponente von Modalität sozusagen in Reinform vertritt, definiert Wahrheit, Notwendigkeit und Möglichkeit auf allen logisch möglichen Welten, was zum einen die Verifikation von Propositionen zu einem praktisch-kommunikativen Unding macht und zum anderen die Tatsache außer Acht lässt, dass Wahrheiten (oder Falschheiten) nie ohne Kontext, sprechende Person und gemeinsame Unterstellungen bei Sprecher und Hörer geäußert werden. Wann haben Sie, Hand auf's Herz, das letzte Mal eine logische Wahrheit der Art „Gestern regnete es oder es regnete nicht“ oder " $2+2=4$ “ gesagt? Oder, mit den Worten Kiefers ausgedrückt: „Clear cases of ,alethically modal' sentences do not occur frequently in everyday discourse." (Kiefer 1994: 2518) Kommunikation baut immer auf einem wissensmässigen Hintergrund auf, Äußerungen, die eines solchen Hintergrunds nicht bedürfen, sind kommunikativ uninteressant oder einfach gesagt langweilig. Wir sind damit bei dem, was man epistemische Modalität genannt hat, also der Art von Modalität, die Notwendigkeit und Möglichkeit auf dem Hintergrund von Wissen analysiert (vgl. Lyons 1977: 793ff., Cann 1993: 279). 
ist nach Cann (1993: 279) nicht als logisch wahr (oder alethisch notwendig, vgl. Lyons 1977: 791) anzusehen, da wir uns durchaus Welten vorstellen können, in denen die Raumtemperatur unter dem Gefrierpunkt oder über dem Siedepunkt von Wasser liegt. Die Interpretation geschieht epistemisch: Die von oben genanntem Satz ausgedrückte Proposition ist wahr g.d.w. Wasser bei Raumtemperatur nass ist in jeder Welt, „[...] consistent with what is (objectively) known about the temperature of rooms and about the properties of water." (Cann 1993: 279) Die Wahrheit solcher Propositionen hängt nicht, wie Cann (1993: 279) richtig bemerkt, von Welten ab, in denen die Räume eine Durchschnittstemperatur von $-50^{\circ} \mathrm{C}$ aufweisen oder Wasser bei $+20^{\circ} \mathrm{C}$ in einen festen Aggregatszustand übergeht.

\subsection{Der Schritt zu relativer Modalität: Evidenzen, Normen und Umstände}

Wie aus obiger informalen Wahrheitsbedingungen hervorgeht, findet sich in formallogischen Ansätzen zur epistemischen Modalität keine Bezugnahme auf den Sprecher und die Evidenz, welche den Bereich der evaluierten Welten einschränkt, er wird als objektiv präsentiert, wie auch Lyons in anderem Zusammenhang hervorhebt (Lyons 1977: 792). Dies hat mit dem Konzept der zugänglichen Welten zu tun, das in der formalen Semantik zur Modellierung epistemischer (und anderer) Modalität herangezogen wird. Diese „Standardanalyse“ zur Modalität, wie sie auch genannt wird (vgl. Kratzer 1991: 642), sei hier nicht dargestellt, zu einer kurzen Darstellung des Anwendungsbereichs zugänglicher Welten vgl. z.B. Cann (1993: 276-281). Stattdessen seien hier die Grundgedanken der formalen Analyse von Modalität von Angelika Kratzer skizziert, wie sie sie in einem kurzen Handbuchartikel dargelegt hat (Kratzer 1991). Das hier vertretene Konzept von Modalität als "relativer Modalität“ ist meiner Meinung nach vorzuziehen, ${ }^{8}$ weil es die linguistisch plausible Annahme von Hintergrundwissen von Modalen mit einer exakten wahrheitsfunktionalen Modellierung verbindet. Ein weiterer Vorzug von Kratzers Ansatz ist, dass der Äußerer modaler Sätze nicht aus der Analyse hinaustheoretisiert wird, wie es in der Standardanalyse oft geschieht. Als letztes ist die Breite des Ansatzes zur relativen Modalität zu nennen, weil hier neben 
epistemischer und deontischer noch ein anderer Typ, die Umstandsmodalität („circumstantial interpretation“, vgl. Kratzer 1991: 640), behandelt wird. Alethische Modalität kommt in Kratzer (1991) bezeichnenderweise nicht zur Sprache; wie ich oben schon angedeutet habe, scheint die rein logische Interpretation von Notwendigkeit und Möglichkeit - in allen möglichen Welten - eine Konstruktion des Logikers zu sein, die in den natürlichen Sprachen kein Gegenstück zu haben scheint (sieht man von „langweiligen Wahrheiten“ einmal ab). Ich halte mich wie gesagt im Folgenden an Kratzer (1991) sowie Chierchia/McConnell-Ginet (1990: 234-241), welche die Grundgedanken Kratzers ihrer Analyse von Modalen zugrundelegen.

In Übereinstimmung mit der logisch-alethischen Standardannahme, dass die Dimensionen Notwendigkeit/Möglichkeit jeglicher Erscheinungsform von Modalität zugrunde liegen („Modality has to do with necessity and possibility“, Kratzer 1991: 639; s.a. Kiefer 1994: 2515 sowie Kiefer 1998: 1), versieht Kratzer modalisierte Sätze mit Paraphrasen, welche die zahlreichen mehrdeutigen modalen Ausdrücke natürlicher Sprachen (z.B. engl. must, can, dt. müssen, können) vereindeutigt und sie genau einer modalen Erscheinungsform zuordnet:

(8a) In Anbetracht der verfügbaren Evidenz: Es ist notwendig/möglich, dass $p$ (epistemische Modalität)

(8b) In Anbetracht der gültigen Normen, Ideale und Gesetze: Es ist notwendig/möglich, dass $\mathrm{p}$ (deontische Modalität)

(8c) In Anbetracht der relevanten Umstände: Es ist notwendig/möglich, dass p (Umstandsmodalität)

Siehe dazu folgendes Beispiel aus dem Deutschen:

(9a) Lise muss bei der Prüfung durchgefallen sein:

(9b) In Anbetracht der verfügbaren Evidenz (sie wirkt niedergeschlagen und weint, ist noch nicht nach Hause gekommen usw.) ist es notwendig, dass sie durchgefallen ist (epistemische Modalität, Notwendigkeit)

Dass die temporal-aspektuelle Morphologie der von Modalverben eingebetteten Verbalkomplexe den jeweiligen modalen Typ (epistemisch, deontisch oder umstandsmodal) steuern kann, wurde bisher nicht hinreichend beachtet (vgl. aber die Überlegungen zum Modalität-Tempus-Zusammenhang in Kiefer 1998: 33 sowie Lyons' These von „Tense as a modality“ in Lyons 1977: 809-823). Der 
Infinitivkomplex durchgefallen sein mit einer wiederum eingebetteten und von sein regierten Partizipform durchgefallen bewirkt eine starke Präferenz der epistemischen Modalität, wie obiger Satz sowie seine Interpretation in der Paraphrase zeigt. Eine Einbettung des infinitivischen Vollverbs direkt unter das selbe Modalverb eröffnet Interpretationen in allen drei modalen Bereichen:

(10a) Lise muss bei der Prüfung durchfallen:

(10b) In Anbetracht der verfügbaren Evidenz (sie hat sich nicht ordentlich vorbereitet, ist schon bei früheren Prüfungen durchgefallen, der Prüfer gilt als streng, das Thema ist formale Semantik usw.) ist es notwendig, dass sie durchfällt (epistemische Modalität, Notwendigkeit)

(10c) In Anbetracht der gültigen Normen (sie hat anstatt der für das Bestehen der Prüfung notwendigen 50 Punkte nur 49 erreicht) ist es notwendig, dass sie durchfällt (deontische Modalität, Notwendigkeit) - hier ist übrigens auch die nur bei deontischer Modalität vorkommende Interpretation als Performativ möglich (vgl. Chierchia/McConnell-Ginet 1990: 237), geäußert z.B. von einem autorativen Prüfer zu seinen Beisitzern vor Lises Prüfung: Es ist dieser Prüfer, der die Normen für die kommende Prüfung setzt.

(10d) In Anbetracht der relevanten Umstände (ihr mentaler Zustand ist derzeit so beschaffen, dass sie die Äußerungen anderer nicht versteht und auch selbst nichts äußern kann; oder: aufgrund einer vorübergehenden Störung vergisst sie alles, was sie gelesen hat) ist es notwendig, dass sie durchfällt (Umstandsmodalität, Notwendigkeit)

Es ist sicherlich eine interessante Frage, ob diese Variation von Verbalmorphologie im eingebetteten Infinitivkomplex in Beziehung zum Modalitätstyp des modalen Gesamtsatzes rein grammatisch bedingt ist oder Ergebnis semantisch-pragmatischer Wechselwirkungen zwischen Modalbedeutung und temporal-aspektuellen Eigenschaften in der eingebetteten Komponente ist. Epistemische, deontische und Umstandsmodalität haben ohne Zweifel temporale Dimensionen, z.B. scheint die deontische Modalität stärker mit zukünftigen oder gegenwärtigen Ereignissen, weniger mit vergangenen, assoziiert zu sein (dies wird durch obige Beispiele zumindest nicht widerlegt). Hier sind sicherlich weitere Untersuchungen nötig.

Es folgen nun einige Beispiele für die Möglichkeitsdimension, wie sie z.B. in der Semantik von kann vorliegt. Auch hier wäre es, nach dem oben Gesagten, notwendig, die Form und Bedeutung der vom Modalverb regierten verbalen Komplemente in ihrer Wirkung auf die Gesamtmodalität zu beachten. Des 
weiteren liegt im Bereich der deutschen Modalverben zusätzlich paradigmatische Variation des Modalverbs vor, insbesondere die modalen Konjunktivvarianten ${ }^{9}$ (z.B. kann - könnte). Auch dies wäre zu berücksichtigen. Wenn ich diese zwei wichtige Punkte nun überspringe, so deshalb, weil sie Kratzers Prinzip, die in den Paraphrasen angedeuteten modalen Hintergründe wahrheitsfunktional zu explizieren, keinen Abbruch tun.

(11a) Lise kann die Prüfung bestehen:

(11b) In Anbetracht der verfügbaren Evidenz (sie ist guten Mutes, hat sich ordentlich vorbereitet, ist selten bei früheren Prüfungen durchgefallen, der Prüfer gilt als gutmütig usw.) ist es möglich, dass sie die Prüfung besteht (epistemische Modalität, Möglichkeit)

(11c) In Anbetracht der gültigen Normen (sie hat eben erst die notwendigen Papiere für die Zulassung zur Prüfung beigebracht) ist es möglich, dass sie die Prüfung besteht (deontische Modalität, Möglichkeit)

(11d) In Anbetracht der relevanten Umstände (sie ist frisch, ausgeruht und motiviert) ist es möglich, dass sie die Prüfung besteht (Umstandsmodalität, Möglichkeit)

Es ist einmal zu bemerken, dass Kratzer (1991) mit der Umstandsmodalität einen Modalitätstyp einführt, der von Modalitätstheoretikern nicht immer zum Kern der Modalitätskategorie gerechnet wird. Die relevanten Umstände der Umstandsmodalität nehmen im Allgemeinen auf permanente oder vorübergehende Fähigkeiten oder Dispositionen Bezug (vgl. die Beispiele oben), die dem modalen Gesamtsatz seine spezielle Prägung verleihen. ${ }^{10}$ In Bezug auf Möglichkeit ist es im Wesentlichen eine generische Interpretation des unmodalen Satzrestes, welche in umstandsmodalen Sätzen mit kann hervortritt. Chierchia/McConnell-Ginet (1990: 234) demonstrieren diesen Zusammenhang an folgenden zwei Sätzen:

(12a) John runs 50 miles without ever stopping

(12b) John can run 50 miles without ever stopping

Im Falle einer generischen Interpretation des modallosen Satzes (seine Wahrheit wird nicht im durch das Präsens indizierten Redezeitpunkt evaluiert) ist der mit can modalisierte zweite Satz eine Paraphrase. Die generische Interpretation der „Umstandsmodalität des Möglichen“ ist wohl eine feste Eigenschaft dieses Modaltyps (vgl. auch Lise kann die Prüfung bestehen oben unter einer 
umstandsmodalen Lesart). Schwieriger ist die „Umstandsmodalität des Notwendigen“ zu charakterisieren. Sie scheint mir in einer streng-kausalen und in diesem Sinne notwendigen Relation zwischen den relevanten Umständen (im obigen Beispiel Lise muss bei der Prüfung durchfallen: Lises mentaler Zustand ist derzeit so beschaffen, dass sie die Äußerungen anderer nicht versteht und auch selbst nichts äußern kann; oder: aufgrund einer vorübergehenden Störung vergisst sie alles, was sie gelesen hat) und der Proposition des unmodalisierten Restsatzes (Lise fällt bei der Prüfung durch) zu bestehen. Es ist leicht zu sehen, dass diese strenge Kausalität bei den anderen zwei Modalitätstypen nicht nachzuweisen ist. Im Falle der epistemischen Modalität der Notwendigkeit in unserem Beispiel Lise muss bei der Prüfung durchfallen sind die hier angeführten Beispiele verfügbarer Evidenz weder notwendig (es könnten auch ganz andere Evidenzen den Äußerer dazu führen, die Notwendigkeit dessen, dass Lise bei der Prüfung durchfällt, zu behaupten, z.B. dass Lise ihm mitgeteilt hat, dass sie absichtlich durchfallen will) noch hinreichend (es könnten alle die angeführten Evidenzen vorliegen, und der Äußerer des Satzes trotzdem die Notwendigkeit dessen, dass Lise durchfällt, zurückweisen):

(13) In Anbetracht der verfügbaren Evidenz (sie hat sich nicht ordentlich vorbereitet, ist schon bei früheren Prüfungen durchgefallen, der Prüfer gilt als streng, das Thema ist formale Semantik usw.) ist es notwendig, dass sie durchfällt (s.a. 10b)

Dass epistemische Modalität und Umstandsmodalität in einem besonderen Verhältnis zueinander stehen, wird auch dadurch erhärtet, dass die Entitäten, welche durch Schlussprozeduren aus epistemischen und umstandsmodalen konversationellen Hintergründen hervorgehen, nicht denselben Status haben. Aus der logischen Modellierung ist dieser Unterschied nicht zu erkennen - er scheint erst auf, wenn wir den Gebrauch von Modalen und ihren Benutzer mit einbeziehen. Kiefer (1994: 2517) zitiert eine frühere Arbeit von Kratzer (Kratzer 1981), wo sie sagt:

„If we use an epistemic modal, we are interested in what may or must be the case in our world, given everything we know already. And if we use a circumstantial modal, we are interested in what can or must happen, given circumstances of a certain kind“ (Kratzer 1981: 52, zit. nach Kiefer 1994: 2517; Hervorheb. im Original). 
Für den Sprecher bedeutet dies, dass die Konklusion aus dem konversationellen Hintergrund im Falle epistemischer Modalität die Wahrheit einer Proposition betrifft („modalities of proposition“, vgl. Kiefer 1998: 11), im Falle der Umstandsmodalität jedoch das Eintreten eines Ereignisses („modalities of events“, vgl. Kiefer 1998: 11; s.a. Kiefer 1994: 2517, der in diesem Zusammenhang auf Perkins 1983: 35 hinweist). Ohne hier in die analytischen Details zu gehen, sei nur festgehalten, dass Kausalität keine Beziehung zwischen Propositionen ist (nur z.B. Implikations- oder Schlussverhältnisse), sondern zwischen Ereignissen (oder Zuständen) in der Welt. Ereignisrelatierte Umstandsmodalität und Kausalität gehen also gut im Tritt, was in unabhängiger Weise die oben vertretene Kausalitätshypothese seitens der Umstandsmodalität stützt.

\subsection{Evaluierung modaler Ausdrücke relativ zu konversationellen Hintergründen}

Ähnliche Überlegungen - allerdings mit verschiedenen Resultaten - ließen sich in Verbindung mit deontischer Modalität anstellen. ${ }^{11}$ Ich möchte aber stattdessen, aus Platzgründen, wieder zu meiner Besprechung von Kratzers Theorie der „relativen Modalität“ zurückkehren. Wie eben ausgeführt, weist sie durch ihr Konzept der Umstandsmodalität (s.a. Kiefer 1998: 9f.) einem weit verbreiteten Gebrauch von Modalverben und modalisierten Sätzen überhaupt einen theoretischen Platz zu. Der andere wichtige theoretische Aspekt ist, dass Modalität in zwei handhabbare Stücke aufgespaltet wird: zum einen die konversationellen Hintergründe („conversational backgrounds“, vgl. Kratzer 1991: 641, „modal base“, vgl. Chierchia/McConnell-Ginet 1990: 237), welche den Modalitätstyp festlegen (epistemisch, deontisch oder umstandsmodal) und in unseren Beispielen informell durch „In Anbetracht ..." gegeben sind; zum anderen das „neutrale“ Modal (Kratzer 1991: 640), das nun eindeutig die modale Dimension Notwendigkeit/Möglichkeit festlegt - und nicht mehr. Das neutrale Modal, das in unseren Beispielsätzen durch „... ist es notwendig, dass ...“ bzw. „... ist es möglich, dass ..." eingeleitet wird, genügt formal den Sätzen der einfachen alethischen Modallogik, wie sie eingangs charakterisiert worden ist. Der einzige Unterschied liegt in der Evaluierungsprozedur bzw. den Wahrheitsbedingungen des modalen Ausdrucks: Die Evaluierung des neutralen Modals (notwendig/möglich) geschieht nun relativ $\mathrm{zu}$ einem kontextuell gegebenen konversationellen Hintergrund, der wiederum durch eine Relative- 
Welten-Semantik modelliert wird. Die formale Umsetzung dieser Ideen im Framework von Kratzer (1991) möchte ich nun ganz kurz andeuten.

In der intensionalen Semantik (vgl. Chierchia/McConnell-Ginet 1990: ch. 5) werden Propositionen mit der Menge der möglichen Welten identifiziert, in denen eine Proposition wahr ist. Umgekehrt: Eine Proposition p ist wahr in einer Welt $\mathrm{w} \in \mathrm{W}$, gdw. $\mathrm{w} \in \mathrm{p}$ (Kratzer 1991: 640). Folgende logischen Beziehungen können mit diesem einfachen Instrumentarium definiert werden (vgl. Kratzer 1991: 641):

\section{(14a) Folgerung}

Eine Proposition p folgt aus einer Menge von Propositionen A gdw. p in allen den Welten von $\mathrm{W}$ wahr ist, in denen alle Propositionen von A wahr sind (Lise besteht die Prüfung, wenn sie "Formal semantics" von R. Cann liest ist wahr, wenn in allen Welten von W, in denen Lise Formal semantics liest, auch die Proposition, dass Lise die Prüfung besteht, wahr ist).

(14b) Konsistenz

Eine Menge von Propositionen A ist konsistent gdw. es eine Welt in W gibt, in der alle Propositionen von A wahr sind (eine Menge von Propositionen A', bestehend aus den beiden Propositionen, dass Lise die Prüfung besteht (p1) und dass Lise die Prüfung nicht besteht (p2), ist in dem uns vertrauten Universum $\mathrm{W}$ nicht konsistent, da $\mathrm{p}$ und $\neg \mathrm{p}$ nicht beide wahr sein können).

(14c) Kompatibilität

Eine Proposition p ist kompatibel mit einer Menge von Propositionen A gdw. die Vereinigung $A \cup\{p\}$ konsistent ist (die Proposition, dass Lise die Prüfung bestanden hat $\left(\mathrm{p}^{\prime}\right)$, ist nicht kompatibel mit der zweielementigen Menge A' von Propositionen, dass Lise 49 Punkte erreicht hat (p1) und das Prüfungsreglement mindestens 50 Punkte zum Bestehen der Prüfung vorschreibt (p2); die Vereinigungmenge $V=A^{\prime} \cup\left\{p^{\prime}\right\}=\{p 1, p 2\} \cup\left\{p^{\prime}\right\}=$ $\left\{\mathrm{p} 1, \mathrm{p} 2, \mathrm{p}^{\prime}\right\}$ ist in dem uns vertrauten Universum nicht konsistent, es sei denn, es gibt eine Welt, in der Lise die zum Bestehen der Prüfung erforderliche Punktzahl nicht erreicht und die Prüfung besteht.

Konversationelle Hintergründe liefern das, was wir wissen (epistemische Modalität), was die Normen vorschreiben (deontische Modalität) oder was die relevanten Umstände sind (Umstandsmodalität), und das für jede mögliche Welt. Formal werden konversationelle Hintergründe als Mengen von Propositionen modelliert, die jeder möglichen Welt durch eine Funktion zugeordnet werden (epistemische, deontische und umstandsmodale Hintergründe sind von Welt zu Welt verschieden). Diese Funktion weist jeder möglichen Welt $\mathrm{w}$ die Menge $\mathrm{p}$ von Propositionen $\mathrm{zu}$, so dass $\mathrm{p}$ in $\mathrm{w}$ gewusst 
wird (epistemische Modalität), die Normen (Ideale, Gesetze) p in w gelten und die relevanten Umstände $\mathrm{p}$ in $\mathrm{w}$ herrschen (Umstandsmodalität). Mit anderen Worten, die Funktion für konversationelle Hintergründe bildet mögliche Welten w auf Mengen von Propositionen ab (so z.B. Chierchia/McConnell-Ginet 1990: 237f.).

An dieser Stelle seien einige kurze Bemerkungen zur Syntax modaler Ausdrücke eingeschoben. Sowohl Kratzer (1991) als auch Chierchia/McConnell-Ginet (1990: 235) analysieren modale Ausdrücke als Satzoperatoren. Im generativ orientierten syntaktischen Rahmen von Chiercia/McConnell-Ginet (1990) werden sie neben Negation sowie verbalen Tempus- und Flexionsmerkmalen im INFL-Knoten generiert (Chiercia/McConnell-Ginet 1990: 235). In der Logischen Form erscheinen Modale wie andere INFL-Elemente dann als dem S-Knoten adjungiert, was die gewünschten Voraussetzungen für eine dem Kompositionalitätsprinzip genügende semantische Interpretation von Modalen als Satzoperatoren schafft. ${ }^{12}$

Sätze mit Modalen werden grundsätzlich auf einem konversationellen Hintergrund ausgewertet. Steht $\alpha$ für irgendeinen Satz und $f$ für irgendeinen konversationellen Hintergrund, ist $[[\alpha]]^{f}$ die Proposition, die von $\alpha$ auf dem Hintergrund von f ausgedrückt wird (Kratzer 1991: 641). Enthält $\alpha$ kein modales Element, hat der Parameter f keine Aufgabe; im Falle eines modalen Ausdrucks jedoch repräsentiert $[[\alpha]]^{\mathrm{t}}$ nur dann eine interpretierbare Proposition, wenn $\mathrm{f}$ den nötigen Hintergrund aus dem Gebrauchskontext liefert. In diesem Fall ist die Interpretation des modalen Ausdrucks entscheidend vom Hintergrund $\mathrm{f}$ abhängig (Kratzer 1991: 641). f wird, wie auch in anderen Ansätzen zur modalen Semantik (vgl. Chierchia/McConnell-Ginet 1990: 237f.), als Funktion $f(w)$ von möglichen Welten w auf Mengen von Propositionen interpretiert.

Sätze mit den Modalen must und can können nun nach dem Gesagten für jeden Satz $\alpha$ und jeden konversationellen Hintergrund f folgendermaßen semantisch interpretiert werden (vgl. Kratzer 1991: 641; s.a. die informelle Darstellung in Kiefer 1994: 2518):

(15a) $[[\text { must } \alpha]]^{f}=\left\{w \in W:[[\alpha]]^{f}\right.$ folgt aus $\left.f(w)\right\}$

(15b) $[[\text { can } \alpha]]^{\mathrm{f}}=\left\{\mathrm{W} \in \mathrm{W}:[[\alpha]]^{\mathrm{f}}\right.$ ist kompatibel mit $\left.\mathrm{f}(\mathrm{w})\right\}$ 
Die Bedeutung des Satzes Lise muss bei der Prüfung durchgefallen sein ([[must $\left.\alpha]]^{[}\right)^{13}$ z.B. ist gleich der Menge von Welten $w$, für die gilt, dass aus alldem, was wir in w wissen $(f(w))$, folgt, dass Lise bei der Prüfung durchgefallen ist $([[\alpha]])$. Wenden wir den Folgebegriff wie oben expliziert auf diese Formulierung an, heißt dies: Die Bedeutung des Satzes ist gleich der Menge Welten w, für die gilt: In allen Welten $w$, in denen die unser Wissen beschreibende Propositionsmenge $f(w)$ wahr ist (d.h. alle in dieser Menge enthaltenen Propositionen sind wahr), ist auch die Proposition $[[\alpha]]^{\uparrow}$ wahr. Der Satz Lise muss bei der Prüfung durchgefallen sein ist damit in einer Welt $\mathrm{w}$ wahr gdw. aus alledem, was wir in $\mathrm{w}$ wissen (Lise wirkt niedergeschlagen und weint, ist noch nicht nach Hause gekommen, die Prüfung ist beendet usw.), folgt, dass Lise bei der Prüfung durchgefallen ist (zu weiteren Beispielen sowie einer kritischen Diskussion der Schluss- und Inferenzverfahren in epistemischen sowie deontischen Interpretationen vgl. Kiefer 1998: 11-13).

In ähnlicher Weise werden Sätze mit können (könnte) interpretiert, vgl. Lise kann/könnte bei der Prüfung durchgefallen sein. Die Bedeutung des Satzes ist gleich der Menge von Welten w, für die gilt, dass die Vereinigungsmenge aus der modallosen Proposition $[[\alpha]]^{\mathrm{f}}$ und der Menge von Propositionen, welche den konversationellen Hintergrund beschreiben $(\mathrm{f}(\mathrm{w}))$ konsistent ist:

(16a) $[[\alpha]]^{f}$ ist kompatibel mit $f(w)$ gdw. $V=[[\alpha]]^{f} \cup f(w)$ konsistent ist (vgl. die Definition von Kompatibilität weiter oben)

(16b) Die Menge $\mathrm{V}$ ist konsistent gdw. es eine Welt gibt, in der alle Propositionen von V wahr sind (vgl. die Definition von Konsistenz weiter oben)

Mit anderen Worten, der Satz Lise kann/könnte bei der Prüfung durchgefallen sein ist wahr gdw. es eine Welt w gibt, in der sowohl die den konversationellen Hintergrund beschreibenden Propositionen wahr sind als auch die unmodalisierte Proposition, die in etwa dem Satzrest Lise ist bei der Prüfung durchgefallen entspricht, wahr ist. Ich denke, auch diese semantische Explikation von Sätzen mit Modalverben geht gut im Tritt mit unserer Intuition modaler Bedeutungen. 
Kratzers Ansatz ist natürlich viel weit reichender als hier dargestellt, u.a. bietet er eine adäquate Modellierung von graduierter Modalität wie sie im Kontrast bei Sätzen wie Lise ist wahrscheinlich bei der Prüfung durchgefallen vs. Lise muss bei der Prüfung durchgefallen sein vs. Lise kann/könnte bei der Prüfung durchgefallen sein zum Ausdruck kommt. Es zeigt sich auch, dass neben dem konversationellen Hintergrund noch ein weiterer vonnöten ist (Stereotypenhintergrund, vgl. Kratzer 1991: 644, Chierchia/McConnell-Ginet 1990: 239), um zu einer deskriptiv adäquaten Analyse modaler Phänomene zu gelangen. Ich möchte hier abbrechen und ein kleines Zwischenresümee ziehen.

\subsection{Schwachpunkte logisch-semantischer Analysen von Modalität}

Einleitend sprach ich von zwei modalen Grundkomponenten, der logischsemantischen und der pragmatischen. Die logisch-semantische Komponente, die Gegenstand der bisherigen Überlegungen war, hat die semantischen Werkzeuge entwickelt, um das an der Oberfläche wahrheitsbedrohende Element der Modalität einer wahrheitsbasierten Analyse zuzuführen, einer Analyse, welche im Großen und Ganzen unserer doch nicht so unscharfen Urteilskraft in Bezug auf modalisierte Ausdrücke entgegenkommt. Die hier besprochenen Ansätze haben Notwendigkeit und Möglichkeit als modale Grunddimensionen in gewissem Sinne rehabilitiert und sie gleichzeitig zwar als notwendige, aber nicht hinreichende Bestimmungsstücke modaler Bedeutungen ausgewiesen. Die alethische Modalität ist - zielt man mit ihr auf eine Analyse natürlicher Sprachen - eine Schimäre des Logikers, welche auf kontextlose absolute Notwendigkeiten und Möglichkeiten baut, die in einem sprachlichen Diskurs selten, wenn überhaupt, eine Rolle spielen (vgl. auch Kiefer 1994: 2518). Epistemische und deontische Modalität tragen zwar eine kontextvermittlende Qualifizierung wissensbezogen/normbezogen in sich, welche sich allerdings in der Hand des formalen Semantikers zu einer irgendwie gegebenen Menge von Wissens- bzw. Normpropositionen formen. Das, was sich sprachlich oder nichtsprachlich, oft unter komplizierten Diskursverhältnissen, zu einem beidseitigen „Wissen“ ausgebildet hat, wird von formalen Semantikern reduziert auf ein „In Anbetracht der verfügbaren Evidenz ..." oder „In the light of what ist known ..." (vgl. hierzu auch Kiefer 1998: 3). In diesem Zusammenhang bemerkt Lyons (1977: 792): 
"[...] there is no reference to the speaker or to the actual drawing of inferences, but only to the evidence which determines the epistemic necessity in question; and this evidence would be treated [vom Logiker. - VE] as something objective."

In der Tat ist der semantisch-logische Ansatz in Bezug auf Modalität alleine nicht ganz zufrieden stellend. Kratzer (1991) z.B. weist, wie oben ausgeführt wurde, dem konversationellen Hintergrund die Rolle $\mathrm{zu}$, a) den Modalitätstyp (epistemisch, deontisch, umstandsmodal) festzulegen und b) die notwendigen Propositionen zu liefern, auf deren Hintergrund die modallose Proposition evaluiert wird. Punkt b) wird meiner Meinung nach zufrieden stellend formal eingelöst; was aber a), den Modalitätstyp, angeht, bleiben einige Fragen unbeantwortet, u.a. welche Eigenschaften eine Propositionsmenge haben muss, um eindeutig einen der drei Modalitätstypen zu kennzeichnen. Es ist in diesem Zusammenhang sicherlich nicht damit abgetan, auf Referenzen auf Wissen (epistemischer Typ), Normen (deontischer Typ) und Fähigkeiten (umstandsmodaler Typ) hinzuweisen. Zum einen ist es schwer, den Typen überhaupt Bereiche zuzuordnen, welche definitorisch zu guten Ergebnissen führen können. Dies gilt auf jeden Fall für den deontischen (Welche Art von Normen? Gesetze? Allgemein anerkannte Sitten? Usw.) und umstandsmodalen Bereich; insbesondere letzterer Typ wirkt in vielerlei Hinsicht inhomogen möglicherweise sogar als unspezifizierte Restklasse. Zum anderen greifen Wissens- und Normenhintergünde ineinander, bestimmtes Wissen kann zu Norm werden - und: Normen müssen im Allgemeinen gewusst werden, bevor man sich ihrer Einhaltung zuwenden kann. Auch Umstandsmodale und epistemische Modalität sind verbunden. Wie ist z.B. das FähigkeitsUmstandsmodal in Jockl can lift the rock (vgl. Kratzer 1991: 640) auseinander zu halten von der epistemischen Interpretation, in der ich den Glauben daran kundtue, dass die Möglichkeit besteht, dass Jockl den Stein hochheben kann? Wie kann ich zwischen Wissen um kausal günstige Zusammenhänge im umstandsmodalen Bereich (in Anbetracht des (nicht übermenschlichen) Gewichts des Stein, des Zustands von Jockls Muskeln usw., vgl. Kratzer 1991: 640) und der Geltung kausal günstiger Umstände alleine (Umstandsmodal) eine klare Scheidelinie ziehen? Es ist für mich schwer zu sehen, wie eine haltbare formale Trennung verschiedener Typen von Hintergründen aussehen sollte. 
Ein weiterer Punkt, der in den bisher dargestellten semantisch-logischen Ansätzen Probleme aufwirft, ist die Frage, wie es zu den konversationellen Hintergründen kommt, die offenbar die Interpretation modaler Ausdrücke in so hohem Grad bestimmen. Formale Ansätze installieren hierzu eine Funktion, die jeder Welt w (bzw. jedem Welt-Zeit-Paar, vgl. Chierchia/McConnell-Ginet 1990: 237f.) eine Menge von Propositionen zuordnet, welche, informal ausgedrückt, die jeweils relevanten Hintergründe in w beschreiben. Dass diese Hintergründe sehr wohl sprachlich oder im Diskurs hergestellt und unterstellt sein können, liegt schon im Begriff „konversationeller“ Hintergrund. Der Sprecher ist in der semantisch-logischen Perspektive auf eine epistemische Propositionsmenge reduziert, die er weiß; den deontischen Normen kann er unterworfen sein („Ich muss jetzt den Saal verlassen."), häufig jedoch weist er andere auf Normen hin („Du musst jetzt den Saal verlassen.“) oder teilt anderen mit, dass in Bezug auf dritte bestimmte Normen herrschen („Er muss jetzt den Saal verlassen.“). Umstandsmodalität sind im Grunde Sprechereigenschaften, denen sich der Sprecher allerdings in keiner Weise bewusst sein muss, es kann sogar sein, dass er keine Ahnung von ihnen hat. Wenn Jockl niesen muss (Beispiel aus Kratzer 1991: 640), hat er wahrscheinlich nicht die geringste Ahnung, welche physiologischen Prozesse diese kausale Notwendigkeit bewirken. Der Sprecher ist also in sehr verschiedener Weise in den drei Modalitäten involviert, einmal als wissendes Subjekt (epistemische Modalität), einmal teilweise als wissendes Subjekt (Ich kann ein deontisches Muss auch verstehen (und befolgen), ohne die relevanten Normen zu kennen) und einmal als Objekt, das einfach bestimmte Eigenschaften, Fähigkeiten hat oder nicht hat bzw. bestimmten kausalen Prozessen unterworfen ist (Umstandsmodalität). Die semantisch-logische Modellierung dieser Zusammenhänge ist $\mathrm{zu}$ rudimentär, um eine adäquate Charakterisierung von Modalität in natürlichen Sprachen zu geben. Hier ist der Punkt, wo der pragmatische Aspekt von Modalität ins Spiel kommt. Auch er ist, wie einleitend ausgeführt, der Wahrheit als grundlegender Kategorie verpflichtet, allerdings Wahrheit auf Seiten des Sprechers, wodurch Wahrheit den Beiton von Aufrichtigkeit bekommt. Der Rest dieses Beitrags ist der Untersuchung des zweiten Aspekts von Modalität gewidmet.

Eine erste Umformulierung des semantisch-logischen Konzepts der Möglichkeit (wahr in mindestens einer möglichen Welt) hin zur sprecherbezogenen pragmatischen Dimension findet sich in Kiefer (1994: 2515): 
„The person who utters the sentence ,It is possible that it will rain tomorrow' does not normally know for sure what the weather will be like tomorrow. That it will rain tomorrow is not excluded, it is one of the possibilities."

Unsicherheit ist kein Konzept, das direkt formalisiert werden kann. Der formalen Semantik steht hier nur der Weg offen, indirekt, ausgehend von einer (womöglich quantifizierbaren) Menge von gewussten Propositionen über Schlussprozeduren zur unmodalisierten Äußerungsproposition ein formalisierbares Maß zu definieren, das Hintergründe in Bezug auf eine Zielproposition hinsichtlich der Stabilität und Robustheit der Schlussprozeduren abstuft. Versuche in dieser Richtung sind in der Tat gemacht worden (vgl. hierzu Kratzer 1991). Es versteht sich aber von selbst, dass auch in diesen Ansätzen wenig vom Sprecher übrig bleibt und viele der oben gestellten Fragen unbeantwortet bleiben.

\section{Pragmatische Aspekte von Modalität}

Schlägt man den Terminus „Modalität“ in linguistischen Handbüchern nach, wird man nicht viel von den bisher behandelten logischen Aspekten dieser Kategorie erfahren, dafür mehr über den „subjektiven“ Aspekt von Modalität, der von vielen älteren Autoren hervorgehoben worden ist (nachzulesen in Lyons 1977: 792f.; vgl. auch Palmer 1986: 16f.). Modalität ist, wie sich bei genauerem Hinsehen zeigt, in der traditionellen Sprachwissenschaft schon immer als pragmatische Kategorie ausgewiesen worden, „[...] welche sich auf die Art und Weise der Stellungsnahme des Sprechers zur Geltung des in einer Äußerung denotierten Sachverhalts bezieht." (Glück 2000: 446; ähnlich auch Bußmann 2002: 438, beide unter dem Stichwort „Modalität“) Die Tradition in der linguistischen Behandlung von Modalität beinhaltet nicht nur eine starke Fokussierung auf die grammatisch-formale verbmorphologische Kategorie des Modus (zu einer Diskussion vgl. Palmer 1986: 21-23), sondern schließt auch Satztypen (in Verbindung mit Satzmodus, vgl. Bußmann 2002: 582) sowie sogar sprechakttheoretische illokutionäre Einheiten wie Behauptung, Frage, Aufforderung (vgl. Glück 2000: 446 und Bußmann 2002: 438) mit ein. Weiterhin ist im traditionellen Paradigma schon vor langem erkannt worden, dass Modalität als universale Bedeutungskategorie in natürlichen Sprachen mit Hilfe der verschiedensten Formmittel und auf verschiedenen sprachlichen Ebenen 
ausgedrückt werden kann, hier u.a. die bekannten morphologischen Verbmodi (z.B. Indikativ, Konjunktiv und Imperativ), Wortbildungsverfahren wie englische -able- und deutsche -bar-Derivationen (wash-able/wasch-bar, solv-able/lös-bar; vgl. Chierchia/McConnell-Ginet 1990: 234), eine Reihe lexikalischer Mittel (z.B. modale Satzadverbien und Modalverben) und auch syntaktische Konstruktionen (Bußmann 2002: 438), welche auf modale Bedeutungen spezialisiert sind. In Anbetracht dieser Vielzahl heterogener Sprachmittel sowie sprachtheoretischer Dimensionen, unter denen modale Phänomene hier subsumiert werden (man denke z.B. an die grammatischen Satztypen vs. sprachliche Handlungstypen der Sprechakttheorie), scheint es schwer, den Sprecher als die modale Zugkraft und den problematisierten Wahrheitsbezug als modale Kerndimensionen wieder zu erkennen. Ich möchte daher im Folgenden näher auf die Handbuchdarstellung von Modalität von Kiefer (1994) eingehen, die einen ersten Ansatz zur Vereinigung sprachlicher (und theoretischer) Diversivität im Modusbereich mit dem semantischen Kern der Moduskategorie anbietet.

\subsection{Satztyp, Satzmodus und Sprechakttheorie}

Kiefer (1994: 2516, 1998: 14) weist auf zwei Arbeiten von C. Bally hin (Bally 1932, 1942), in denen Bally vorschlägt, Modalität mit der sprecherseitigen Bewertung eines Sachverhaltes zu identifizieren. Demnach besteht jeder Satz aus zwei Bestandteilen, dem was gesagt wird, das Diktum, und dem, wie es gesagt wird, der Modus. ${ }^{14}$ Jener Modus trägt der oben genannten sprachlichen Formvielfalt modalitätstragender Ausdrücke Rechnung, enthält aber, auf Grund des Bewertungskriteriums, auch Ausdrücke, die nicht von allen Grammatikern unmittelbar der Modalität zugerechnet würden (z.B. I think/hope in I think/hope, it is raining, vgl. Kiefer 1994: 2516).

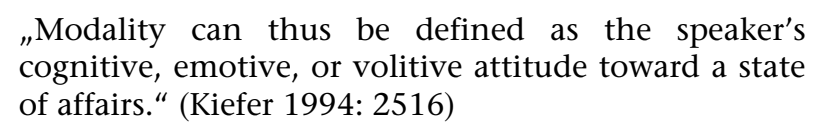

Modalität ist sprecherseitige Einstellung, und die Aufgabe des Linguisten besteht darin, in jedem Satz das Diktum vom modalen Einstellungsausdruck zu trennen. Hat diese Trennung ein Pendant in syntaktischer Zweigliedrigkeit (Matrixausdruck: Einstellungsausdruck, eingebetteter Ausdruck: Diktum), liegt also ein ikonischer Parallelismus der semantischen und der syntaktischen Ebene vor, können die beiden Satzbestandteile relativ einfach auseinander gehalten 
werden. Neben dieser in der syntaktischen Hierarchie verankerten ModusDiktum-Unterscheidung kann der modustragende Ausdruck auch Teil eines einfachen Satzes sein, wo er als Adverbial (probably in It is probably raining), Modalverb (must in It must be raining) oder als Verbflexiv auftreten kann (vgl. Kiefer 1994: 2516).

Mit der Idee der Trennung von Modus und Diktum ist nach Kiefer (1994: 2516), allerdings mehr implizit als explizit, das Verhältnis zwischen Satztypen und Satzmodus verbunden. Satztypen sind in der deutschen Grammatik Aussage-, Frage-, Aufforderungs- Wunsch- und Ausrufesätze (vgl. Bußmann 2002: 582), die - trotz der Sprechhandlungen indizierenden Namen - festgefrorene syntaktische Konstruktionen sind und als grammatisch definierte Formtypen zunächst nicht mit Bedeutungs- oder Funktionsgruppen identifiziert werden dürfen. Satzmodus ist der "modale Wert" von Satztypen (Kiefer 1994: 2516), also im Grunde eine Funktion, welche formal bestimmte Klassen von Sätzen (Satztypen) auf pragmatische „Funktionstypen “15 (Bußmann 2002: 582) abbildet, hierunter Deklarative, Imperative, Interrogative, Optative und Exklamative; das Abbildungsverhältnis braucht nicht eindeutig zu sein (vgl. Kiefer 1994: 2516). Satzmodus wird im Allgemeinen, wie Kiefer (1994: 2516) nun fortfährt, mit Sprechereinstellungen gleichgesetzt, die er mit Hilfe von Paraphrasen verdeutlicht:

(17a) „Der Sprecher nimmt es für gegeben, dass ...“: Deklarativ - z.B. Aussagesätze

(17b) „Der Sprecher möchte wissen, ...“: Interrogativ - z.B. Fragesätze

(17c) „Der Sprecher ist erstaunt darüber, dass ...“: Exklamativ - z.B. Ausrufesätze

(17d) „Der Sprecher möchte den Hörer dazu bringen, dass ...“: Imperative - z.B. Aufforderungssätze

(17e) „Der Sprecher wünscht, dass ...“: Optativ - z.B. Wunschsätze

Ich habe bisher wenig zur deontischen Modalität gesagt, dem modalen Typ also, der mit Verpflichtung und Erlaubnis zu tun hat (vgl. z.B. Lyons 1977: 823ff. sowie Kiefer 1998: 3f., 8f.). Hier, wo plötzlich Sprechakte (vgl. Searle 1971) ins Spiel kommen und mit modalen Kategorien relatiert - wenn nicht identifiziert werden, ist der richtige Platz, ein paar Worte zum deontischen Typ zu sagen. Es besteht im Bereich der deontischen Modalität eine Beziehung zu den alethischen 
Grunddimensionen Sicherheit, Notwendigkeit und Möglichkeit in der Weise, dass „alethische Ausdrücke“ (z.B. müssen, notwendig sein, können, möglich sein) in den meisten Sprachen auch für die Kategorien Verpflichtung und Erlaubnis verwendet werden (Kiefer 1994: 2518). Kiefer schlägt in diesem Zusammenhang eine Arbeitsteilung zwischen Semantik und Pragmatik vor, ${ }^{16}$ wo deontische Notwendigkeit sowie Möglichkeit als semantische Begriffe von Verpflichtung und Erlaubnis als dem pragmatischen Bereich zugehörig abgetrennt werden (Kiefer 1994: 2518 sowie Kiefer 1998: 26f.; zu einer knappen Explikation der Semantik-Pragmatik-Trennung vgl. Kiefer 1998: 2). Jemandem eine Verpflichtung auferlegen oder eine Erlaubnis erteilen sind Sprechakte (Kiefer 1994: 2518):

„By uttering the sentence ,You can park your car here' the speaker states a deontic possibility, but this sentence counts as a speech act of granting permission only if some additional conditions are met (For example, the speaker must have the authority to grant such a permission.)“

Dieser Gedankengang könnte auch auf den Ausdruck epistemischer Modalität ausgeweitet werden. Auch hier werden zunächst die semantischen Wahrheitsbedingungen für "epistemische Notwendigkeit bzw. Möglichkeit“ abgearbeitet, wobei der syntaktische Rahmen prototypisch ein Aussagesatz ist (s.o.). Als welcher Sprechakt könnte ein solcher epistemisch-modaler Aussagesatz zählen? Eine mögliche Antwort gibt Kiefer indirekt in obigen Paraphrasen für Sprechakttypen samt deren Kopplung an grammatische Satztypen: Das, was deklariert wird, ist wiederum der epistemisch-modale Satz, z.B. für Lise kann die Prüfung bestanden haben: „Der Sprecher nimmt es für gegeben, dass Lise die Prüfung bestanden haben kann“. Die Äußerung des Satzes zählt also als Deklaration einer epistemischen Möglichkeit. ${ }^{17}$

Akzeptiert man nun die sprechakttheoretische Auflösung deontischer Modalität, wie sie Kiefer oben vorschlägt, und folgt man meiner Hypothese, dass die Teilung in semantischen (logische Notwendigkeit/Möglichkeit) und pragmatischen Bedeutungsaspekt (Sprechakttyp des Satzes, „X zählt als Y“) nicht auf deontische Sätze (oder: Verwendungen?) beschränkt ist, kann die obige Paraphrase des Deklarativs nicht richtig sein. In Kiefers Beispiel der Erteilung einer Parkerlaubnis durch einen Satz mit dem deontischen (deontisch 
verwendeten) Modal kann handelt es sich ja nicht um den modalisierten Ausdruck, der in die Paraphrase des Erlaubnissprechakts eingebettet ist $\left({ }^{*}\right.$ „Der Sprecher gibt dem Hörer die Erlaubnis, den Wagen hier parken zu können“), sondern um den unmodalisierten Satzrest, der von der semantischen Komponente bereits abgearbeitet worden ist („Der Sprecher gibt dem Hörer die Erlaubnis, den Wagen hier zu parken“), wobei das Modal lediglich den UnterSprechakttyp bestimmt (kann: „Der Sprecher gibt dem Hörer die Erlaubnis, ...", deontische Möglichkeit - muss: „Der Sprecher fordert den Hörer (bestimmt) dazu auf, ...", deontische Notwendigkeit).

Wie könnte eine Rekonstruktion der Bedeutung von epistemisch-modalen Aussagesätzen (bzw. epistemischen Satzverwendungen) nach dem selben Verfahren aussehen? Zunächst wird in eine Sprechaktparaphrase SAP nicht, wie oben, der modale Gesamtsatz eingebettet, sondern - parallel zur Deontik - der unmodalisierte Satzrest (Lise kann die Prüfung bestanden haben: „SAP, dass Lise die Prüfung bestanden hat"). Die Sprechaktparaphrase SAP beinhaltet eine Distanzierung vom Wahrheitswert wahr, vom Zutreffen des vom unmodalisierten Satzrest denotierten Ereignisses in der Äußerungssituation seitens des Sprechers. Diese pragmatische Distanzierung vom Wahrheitswert unterliegt einer Graduierung, deren Parameter von der semantischen Komponente geliefert werden - ähnlich den deontischen Verwendungen, wo die Eingangsbedingungen für den pragmatischen Unter-Sprechakttyp vom Modal abhängig sind (kann: „Der Sprecher distanziert sich stark vom Zutreffen, dass ...“, epistemische Möglichkeit - muss: „Der Sprecher distanziert sich schwach vom Zutreffen, dass ...", epistemische Notwendigkeit). Diese Analyse, ${ }^{18}$ die sicherlich noch weiter begründet werden müsste, scheint mir nicht nur intuitiv mehr einleuchtend (also unser Wissen über Bedeutungen modaler Sätze und deren Gebrauch gut wiedergebend), sie steht meiner Meinung auch gut im Einklang mit der hier verfolgten These des inhärent Unwahrhaftigen in der Modalität, die auch in folgendem Zitat von Palmer (1986: 51) zum Ausdruck kommt:

„There are at least four ways in which a speaker may indicate that he is not presenting what he is saying as a fact, but rather:

(i) that he is speculating about it

(ii) that he is presenting it as a deduction

(iii) that he has been told about it 
(iv) that it is a matter only of appearance, based on the evidence of (possibly fallible) senses."

Dies habe ich mit "Distanzierung" in meiner obigen Rekonstruktion gemeint: die Möglichkeit des Sprechers, sich indirekt - also nicht über die deklarative Behauptungskomponente - der kategorialen Verpflichtung auf die Faktizität der Proposition zu entziehen, indem er die ja/nein-Kategorisierung in eine flexible Skala unterschiedlicher Sicherheitswerte uminterpretiert. Dies führt hin zum modalen Phänomen der Evidentialität (vgl. Kiefer 1998: 7), der Kennzeichnung der Quelle einer Proposition (vgl. iii) und iv) oben), welche indirekt höchstwahrscheinlich durch praktisch-pragmatische Schlussprozesse - zu einer Interpretation der Distanzierung von der Geltung eines Sachverhaltes beiträgt. Zur Evidentialität folgt weiter unten mehr.

\subsection{Die schwierige Verheiratung von Pragmatik und Logik/Semantik in der modalen Analyse}

Kiefer schließt seine Überlegungen ab mit folgendem „Glaubensbekenntnis“, das wohl den Bogen zur semantisch-formalen Ausgangscharakterisierung von Modalität schlagen soll:

"The view of modality in terms of speaker's attitudes
is consistent with the characterization of modality as
,envisaging several possible courses of events' or
,considering the possibility of things to be
otherwise'." (Kiefer 1994: 2516)

Kiefers Charakterisierung von Modalität gibt, meine ich, gut den traditionellen Standpunkt gegenüber Modalität wider. Wie allerdings das semantisch-logische Konzept von Modalität mit den hier skizzierten Auffassungen einer sprecherorientierten, „pragmatischen“ Modalität in Einklang gebracht werden kann (vorausgesetzt, dies ist notwendig oder wünschenswert), ist alles andere als klar. Obwohl Kiefer in obigem Zitat die Vereinbarkeit der beiden Modalitätskonzepte offenbar als gegeben ansieht, bleibt doch zu fragen, wie und warum. Die Anspielung auf die Mögliche-Welten-Semantik („several possible courses of events", possibility of things to be otherwise") beantwortet diese Frage auf jeden Fall nicht. Auch an dieser Stelle kann dies nicht geleistet werden, alleine deshalb, weil Kiefers eigene Darstellung $\mathrm{zu}$ wenig explizit und allzu 
kursorisch ist. Ich muss es deshalb bei einigen wenigen, sehr spekulativen Zwischenbemerkungen belassen.

Der Gedanke von Diktum und Modus kehrt in der formalen Semantik von Modalität wieder in Form der (modallosen) Satzproposition und der modalen Operatoren. Diese Parallelität deutet an, dass Diktum und Modus im Grunde genommen als semantische Begriffe zu deuten sind, wenn auch teilweise den Eindruck erweckt wird, es handelte sich um syntaktische Konstituenten („[...] each sentence consists of two parts $[\ldots]^{\prime \prime}, \ldots[\ldots]$ the dictum can be identified as ,It is raining' [...]“, vgl. Kiefer 1994: 2516). Ballys Modus wird wie in der formalen Semantik als Satzoperator aufgefasst (vgl. Chierchia/McConnell-Ginet 1990: 235), welcher die Satzproposition modifiziert. Während die modalen Satzoperatoren $\square$ und $\diamond$ in dem Sinne als bedeutungsarm aufgefasst werden, dass sie lediglich die Interpretation der in ihrem Fokus stehenden Satzproposition in quantifikatorischer Weise (alle/mindestens ein) auf mögliche Welten relativieren, ist der Ballysche Modus dagegen ein Konglomerat konglomerierter Einstellungsprädikate, die analytisch selbst wieder in die eigentlichen einstellungsdenotierenden Komponenten und den lexikalischen Bedeutungsrest (vgl. hoffen, erstaunt sein, wünschen ...) geteilt werden müssten. $\mathrm{Zu}$ fragen wäre dann, welcher Art diese rein einstellungsdenotierende Funktion sein sollte. Es ist in der Tat schwer, Pendants in den bisher besprochenen Modellierungen von Modalität zu finden.

Es bleibt weiterhin im Dunkeln, wie Notwendigkeit und Möglichkeit als modale Grunddimensionen in diese Konzeption eingehen können; Kiefer geht auf diesen Punkt nicht ein. $\mathrm{Zu}$ fragen ist auch, wieweit das Einstellungskonzept geeignet ist, Modalität als semantische Grundkategorie zu fundieren. Die von Kiefer gewählten Beispiele legen mit der 1. Person Singular (I think it is raining) einen immanenten, einstellungsrelatierten Sprecherbezug nahe. Konstatierungen in der 3. Person jedoch (He thinks it is raining) führen zum Schluss, dass hier eine Einstellung wie „Der Sprecher nimmt es für gegeben, dass ... “ für alle Deklarative im Spiel sein muss (siehe oben). Think ist also einmal Ausdruck für einen Modaloperator (Matrixsubjekt in der 1. Person) und einmal ein Teilausdruck innerhalb der Satzproposition (Matrixsubjekt in der 3. Person). Und was ist mit Prädikaten wie "Es ist gut, dass ..."? In gewissem Sinne handelt es sich hier 
durchaus um Einstellungen, jedoch Einstellungen, die mit unserem Begriff von Modalität nur schwer in Einklang zu bringen sind.

Damit steht ein weiterer Punkt in Zusammenhang, nämlich die Tatsache, dass ein Teil modaler Verwendungen mit dem Einstellungskonzept von Modalität nicht vereinbar sind. Lyons (1977: 798), der in die modalen Typen eine weitere Subklassifizierung nach den Parametern subjektiv/objektiv einführt, gibt folgendes Beispiel für die objektive Variante epistemischer Modalität anhand des Satzes

\section{(18) Perhaps Alfred is unmarried}

Alfred ist in einer Gruppe von 90 Menschen und wir wissen, dass 30 davon unverheiratet sind (wir wissen aber nicht, wer verheiratet bzw. unverheiratet ist). Die Äußerung des obigen Satzes ist epistemisch objektiv, sie ist ein Fakt, der mit einer gewussten Wahrscheinlichkeit von 1/3 vorgebracht werden kann und in keiner Weise irgendeine „subjektive“ Einstellung des Sprechers enthält. Objektive Verwendungen modaler Sätze sind also, wenn man die subjektiv/objektiv-Unterscheidung akzeptiert, Gegenbeispiele zur einstellungsrelatierten Konzeption von Modalität. ${ }^{19}$

Überhaupt ist der hier vertretene Modalitätsbegriff extrem breit, ${ }^{20}$ die modale Einbettung von Satzpropositionen ist eine Eigenschaft aller Sätze einer Sprache, unbesehen dessen, ob ein modaler Ausdruck in der Oberflächenstruktur vorhanden ist und unbesehen der Bedeutung von Modalität, die sie von anderen semantischen Kategorien im Sprachsystem unterscheiden kann. Diese Allgegenwärtigkeit von Modalität in der Grammatik ist nur zu sichern, wenn Einstellungen auf universale Sprechhandlungen abgebildet werden, was Kiefer letzten Endes auch tut. Obwohl der Zusammenhang zwischen Einstellung, Satzmodus, Satztyp und Sprechakttypen bei Kiefer nicht klar wird, könnte die Relatierung von modalen Typen mit Sprechhandlungen etwas für sich haben. Sprechhandlungen sind sowohl sprachstruktur- als auch kontextabhängig und basieren, wie Searle gezeigt hat, auf sprecherseitigen Wahrheits- und Aufrichtigkeitsbedingungen, welche eine Modellierung in einer MöglichenWelten-Semantik verlangen. Eine Identifizierung von Modus mit Sprechakttypen hat jedoch die Eliminierung eines der beiden zur Folge, was weit reichende und 
unerwünschte Folgen haben würde. Eliminiert man z.B. Modus, müsste eine Analyse von Modalverben oder Modaladverbien ausschließlich in Sprechaktkategorien erfolgen (dies hat meiner Kenntnis nach noch niemand vorgeschlagen). Interessanter dagegen ist, die beiden Kategorien aufrechtzuerhalten und stattdessen ihren engen sprachtheoretischen Zusammenhang zu erforschen. Dies ist meines Wissens auch der Weg, den die moderne, pragmatisch ausgerichtete Grammatikforschung geht.

\subsection{Evidentialität}

Es sieht in der Tat so aus, dass logisch-semantisch orientierte Theorien zur Modalität es schwer haben, den pragmatischen Sprecheraspekt, also gewissermaßen den Sprung von der Wahrheit zur Wahrhaftigkeit und Aufrichtigkeit, nachzuvollziehen. Formale Theorien bleiben im Wesentlichen den Polen nicht-möglich vs. notwendig verhaftet, wobei der Zwischenbereich es handelt sich hier um Duale - lediglich durch Mögliches und NichtNotwendiges weiter strukturiert wird. Unsicherheit des Sprechers in Bezug auf den Wahrheitswert der geäußerten Proposition, ein ohne Zweifel zentrales modales Konzept, das auch mit den erwähnten Einstellungskomponenten von Modalität sehr gut im Tritt geht, scheint in logisch-semantischen Ansätzen, wenn überhaupt, nur sehr vermittelt auf. Eine modale Kategorie, welche auf die sprecherseitige Relativierung zur geäußerten Proposition geradezu spezialisiert ist, ist (epistemische) Evidentialität (vgl. hierzu Kiefer 1994: 2517f. sowie 1998: 15-17). Evidentialität kodiert die Quelle der Information, aus welcher der Sprecher Kenntnis über eine Proposition erlangt hat; die syntaktische Kopplung eines Evidentialitätsformativs an den Propositionsausdruck ergibt so eine komplexe Mitteilungseinheit, in welcher der Sprecher dem Hörer gegenüber seine Verantwortlichkeit in Bezug auf die Wahrheit der Proposition relativiert, man könnte auch sagen, „objektiviert“, denn ein Evidentialitätsformativ ist nichts anderes als eine Versprachlichung der Quelle, was in der Kombination mit einer Proposition $\mathrm{p}$ als „Ich habe gesehen/nur gehört (und nicht gesehen)/Hinweise darauf/von einem Dritten erfahren, dass p“ paraphrasiert werden kann. Es gibt Sprachen, in denen alle Sätze eine formale Markierung der Evidentialität erfordern, wo also ein voll grammatikalisiertes Evidentialitätssystem vorliegt (vgl. Kiefer 1994: 2517, Palmer 1986: 27, 51ff.). Im Deutschen z.B. ist dies nicht der Fall (vgl. hierzu Bußmann 2002: 206; Kiefer 1998: 17, Palmer 1986: 71-74), nur einige wenige spezialisierte Konstruktionen 
sowie eine beschränkte Auswahl von Evidentialitätsbedeutungen (v.a. Quotativ, s.u.) stehen dem Sprecher des Deutschen zur Verfügung, die Quelle der Information zu spezifizieren, und damit indirekt die Geltung der Proposition sowie seine Bereitschaft, für die Wahrheit der Proposition einzustehen, zu relativieren (Beispiele aus Bußmann 2002: 206):

(19a) Die Regierung sei nicht zu Verhandlungen bereit (Quotativ ohne Nennung der Quelle; Konjunktiv Präsens)

(19b) Philip will ein direkter Nachfahre Karls des Grossen sein (Quotativ mit Nennung der Quelle (Denotat des syntaktischen Subjekts); Konstruktion mit wollen)

Keines der hier betrachteten formalen Systeme erlaubt, Evidentialität als Unterart epistemischer Modalität auszuweisen. Kratzers Konstrukt konversationeller Hintergründe ist in dieser Hinsicht zu wenig ausgebaut, wie weiter oben schon ausgeführt, ist schon die Trennung der drei modalen Typen epistemisch, deontisch und umstandsmodal anhand der Hintergründe problematisch. Die Menge $\mathrm{H}$ der Propositionen, welche einen aktuellen epistemischen konversationellen Hintergrund beschreiben, könnte im Falle von Evidentialität eine ausgezeichnete Menge von Propositionen $\mathrm{H}_{\mathrm{ev}}, \mathrm{H}_{\mathrm{ev}} \subset \mathrm{H}$, enthalten, welche die jeweilige Quelle der zu kommunizierenden Proposition in einer Weise charakterisieren, die relevant für die Semantik evidentieller Formative ist (direkte visuelle/auditive Evidenz, inferentiell usw., vgl. oben). Ein wie auch immer geartetes Semantikmodul ordnet dann $\mathrm{H}_{\mathrm{ev}}$ den jeweiligen Evidentialitätstyp zu, der z.B. als semantisches Merkmal im Lexikon das dem Typ entsprechende Formativ selegiert. Dieses Gedankenexperiment setzt voraus, dass ein epistemisch modaler Satz zweifach modal gekennzeichnet sein kann, einmal, wie oben demonstriert, evidentiell relativ $\mathrm{zu}_{\mathrm{ev}}$, und einmal entlang der Dimensionen Notwendigkeit und Möglichkeit relativ $\mathrm{zu}$ einem konversationellen Hintergrund, der durch die Komplementärmenge $\mathrm{H}-\mathrm{H}_{\mathrm{ev}}=\mathrm{H}_{\mathrm{al}}$ beschrieben ist. Dass es solche Sätze gibt, legt die Existenz starker und schwacher Inferentiale im Deutschen nahe, welche neben der evidentiell-inferentialen Komponente (,Aus allem, was mir bekannt ist, schließe ich, dass ...') eine Interpretation entlang der Dimensionen notwendig/möglich erfordern, die sich in einer Alternation der beiden modalen Verben muss/dürfte niederschlägt (Beispiel aus Bußmann 2002: 206): 
(20a) Hierbei muss es sich um eine Verwechslung handeln (Inferential, stark)

(20b) Hierbei dürfte es sich um eine Verwechslung handeln (Inferential, schwach)

Auch im Bereich des deutschen Quotativs lässt sich ansatzweise diese Doppelmodalität erkennen. Ein Satz wie

\section{(21) Die Regierung müsse/könne zurücktreten}

signalisiert morphologisch durch den Konjunktiv an den präsentischen Stämmen der Modalverben müssen und können (müss-e, könn-e) die Evidentialität des Quotativs, gleichzeitig wird eine Menge von konversationellen Hintergründen evoziert, relativ zu denen es einmal notwendig (müsse), einmal möglich (könne) ist, dass die Regierung zurücktritt. ${ }^{21}$

Ich meine, all dies bestätigt zwar die Nützlichkeit und Angemessenheit des semantisch-logischen Konzepts konversationeller Hintergründe, macht aber gleichzeitig deutlich, dass aus der Sicht des Linguisten (und diese Perspektive haben wir hier eingenommen), der von sprachlichen Fakten und sprachlicher Diversität ausgeht und semantische Einheiten oft aus den grammatischen Gegebenheiten einer Einzelsprache rekonstruiert, das logische Instrumentarium häufig zu grobmaschig erscheint. Evidentialität ist eine zentrale modale Kategorie, welche die Verpflichtung des Sprechers auf die Wahrheit der Proposition durch eine Differenzierung der Quelle relativiert; eine formale Logik, die ausschließlich auf Notwendigkeit und Möglichkeit (relativ zu Hintergründen) basiert, tut sich verständlicherweise mit dieser „Quellensemantik“ schwer.

\section{ZUM SCHLUSS}

Ich habe in diesem Beitrag versucht, einige sehr grundlegende Fragen und Probleme, die mit Modus und Modalität verbunden sind, so klar wie möglich zu formulieren und in einen Zusammenhang zu stellen, der die Einheitlichkeit und Integrität von Modalität als semantisch-pragmatischer Kategorie wahren kann. Antworten und Lösungen konnte ich, wenn überhaupt, nur andeuten, obwohl ich versucht habe, genügend konkret zu sein, um die Konturen des jeweils ersten Schritts in die richtige Richtung zu zeichnen. Viele der neueren und interessanten Konzeptionen zur Modalität, die auch viel stärker, als ich es hier konnte, in der Analyse einer Einzelsprache verankert sind, habe ich hier nicht 
würdigen können. So z.B. die gründliche Arbeit von Hestbæk Andersen/Smedegaard (2005), wo die Autoren mit dem Werkzeug der systemisch-funktionellen Grammatik Modalität im Dänischen als „zentrale interpersonelle Ressource" analysieren. Ein weiterer wichtiger Aspekt von Modalität, den ich nicht erwähnen konnte, ist die interaktive Genese von Modalität in Diskursen, wo der Wahrheitswert einer Proposition zur Diskussion steht und erst im Verlaufe einer Sequenz quasi verhandelt wird (vgl. Spenader 2004). Ein Punkt, den ich zwar angesprochen habe, aber nicht vertiefen konnte, ist, dass modale Einheiten stark dazu tendieren, mit anderen Sprachkategorien einer Einzelsprache zu interagieren, teilweise deren Funktionen zu übernehmen und syntaktisch-morphologischen Kombinationsrestriktionen unterliegen. Arndt (2003) ist eine solche Analyse, wo die Beziehungen zwischen Tempus, Aspekt und Modalität untersucht werden und gezeigt wird, dass hier keine einfache Relation zwischen Ausdruck und Inhalt besteht. In diesem Zusammenhang ist auch Engerer (2005) zu sehen, wo die Tradition, Modalität als Bedeutungsmerkmal in die deutsche Tempussemantik zu integrieren, angegriffen und ein Vorschlag $\mathrm{zu}$ dessen Eliminierung vorgebracht wird. Eine repräsentationelle Theorie der Sprachstruktur wird in Thrane (2004) entwickelt, in die Modalisierung neben der Informations- und Argumentstruktur als wichtige Repräsentationsebene eingeht.

Problematisierte Wahrheit und Wahrhaftigkeit in Bezug auf eine geäußerte Proposition dienten als Richtschnur durch den Dschungel verschiedener Ansätze, Modalität einer sprachgerechten Analyse zuzuführen. Ich habe auch versucht, die Kluft zwischen logisch-philosophischen und linguistischen Theorien zur Modalität ansatzweise zu überbrücken, es ist immer noch, auch nach Abschluss dieser Arbeit, meine Überzeugung, dass es keine „logische“ und "linguistische Modalität“ geben kann, beide Disziplinen haben dasselbe Phänomen vor Augen. Meine hier vorgelegte Diskussion hat für mich - und ich hoffe auch, für den Leser - in schlagender Weise gezeigt, wie sehr Sprache, sprachliche Kategorien sowie Sätze und deren Verwendungen auf Wahrheitsbezug und Aufrichtigkeit beim sprachlichen Handeln getrimmt sind. Modalität, wo Wahrheitswerte plötzlich in empirisch und in der Äußerungssituation nicht direkt zugänglichen „Welten“ evaluiert werden müssen, wo man etwas behauptet - aber gleichzeitig einräumt, es könnte auch anders sein, ist meiner Meinung nach das sprachliche Mittel, welches es dem 
Sprecher ermöglicht, zeitweise aus diesem Wahrheitskorsett auszubrechen und gleichzeitig seine Integrität als glaubhafter, moralisch verantwortlicher Agent zu bewahren. Eine grammatische Kategorie der Lüge oder eine konventionalisierte Sprechhandlung dieser Art kann es daher nicht geben.

\section{LITERATUR}

Arndt, Hans (2003), „Time, tense and other matters“, in: Engerer, Volkmar (red.) (2003), Tid i sprog, Århus: SB-Tryk, pp. 105-117 (= Tidsskrift for Sprogforskning, 12003$)$.

Bally Charles (1932), Linguistique générale et linguistique française, Paris: Leroux.

Bally, Charles (1942), „Syntaxe de la modalité explicite“, Cahiers Saussure, 2 (1942), pp. 3-13.

Bußmann, Hadumod (2002), Lexikon der Sprachwissenschaft, Stuttgart: Kröner.

Borsley, Robert D. (1997), Syntax-Theorie. Ein zusammengefasster Zugang, Tübingen: Niemeyer.

Cann, Ronnie (1993), Formal semantics, Cambridge: Cambridge University Press.

Chierchia, Gennaro, Sally McConnell-Ginet (1990), Meaning and Grammar. An Introduction to Semantics, Cambridge/Mass., London: The MIT Press.

Davies, M. (1994), „Modal Logic“, in: Asher, R. E. (Editor-in-Chief) (1994), The Encyclopedia of Language and Linguistics, Vol. 5, Oxford etc.: Pergamon, pp. 2508-2515.

Dowty, David R., Robert E. Wall, Stanley Peters (1981), Introduction to Montague semantics, Dordrecht: Reidel.

Eisenberg, Peter (1999), Grundriss der deutschen Grammatik, Bd. 2, Der Satz, Stuttgart, Weimar: Metzler.

Engel, Ulrich (1988), Deutsche Grammatik, Heidelberg: Groos.

Engerer, Volkmar (2005), „Bedeutungsminimalismus und Temporalsemantik. Zum Status zeitlicher und nicht-zeitlicher Bedeutungskomponenten in der Analyse der deutschen Tempora", in: Cecchini, Leonardo, Alexandra Kratschmer (eds.) (2005), Ancora un'ultima occhiata ... La virtù della ponderatezza. Festskrift $i$ anledning af Svend Bachs 60 års fødselsdag, Århus: Aarhus Universitet, $20-41$. [www.hum.au.dk/cfk/pages/publications/lc_ak/festskrift_svendbach.htm]

Glück, Helmut (2000), Metzler Lexikon Sprache, Stuttgart: Metzler.

Heidolph, Karl Erich, Walter Flämig, Wolfgang Motsch (1981), Grundzüge einer deutschen Grammatik, Berlin: Akademie-Verlag.

Hestbæk Andersen, Thomas, Flemming Smedegaard (2005), „Interpersonel farvning i dansk“, Tidsskrift for Sprogforskning, 3/1 (2005), pp. 5-27.

Hindelang, Götz (1994), Einführung in die Sprechakttheorie, Tübingen: Niemeyer.

Kempson, Ruth M. (1977), Semantic Theory, Cambridge etc.: Cambridge University Press. 
Kiefer, Ferenc (1994), „Modality“, in: Asher, R. E. (Editor-in-Chief) (1994), The Encyclopedia of Language and Linguistics, Vol. 5, Oxford etc.: Pergamon, pp. 2515-2520.

Kiefer, Ferenc (1998), „Modality“, in: Verschueren, Jef, Jan-Ola Östman, Jan Blommaert, Chris Bulcaen (eds.) (1998), Handbook of Pragmatics, Amsterdam, Philadelphia: Benjamins, pp. 1-36.

Kratzer, Angelika (1981), „The notional category of modality“, in: Eikmeyer, Hans-Jürgen, Hannes Rieser (eds.) (1981), Words, Worlds, and Contexts. New Approaches in Word Semantics, Berlin: de Gruyter, pp. 38-74.

Kratzer, Angelika (1991), „Modality“, in: von Stechow, Arnim, Dieter Wunderlich (Hgg.) (1991), Semantik. Ein internationales Handbuch der zeitgenössischen Forschung, Berlin: Walter de Gruyter, pp. 639-650.

Levinson, Stephen C. (1990), Pragmatik, Tübingen: Max Niemeyer Verlag.

Lohnstein, Horst (1996), Formale Semantik und natürliche Sprache, Opladen: Westdeutscher Verlag.

Lyons, John (1977), Semantics, Vol. 2, Cambridge: Cambridge University Press.

Palmer, F. R. (1986), Mood and Modality, Cambridge etc.: Cambridge University Press.

Perkins, Michael R. (1983), Modal Expressions in English, London: Frances Pinter.

Rolf, Eckard (1997), Illokutionäre Kräfte. Grundbegriffe der Illokutionslogik, Opladen: Westdeutscher Verlag.

Searle, John R. (1971), Sprechakte. Ein sprachphilosophischer Essay, Frankfurt/M.: Suhrkamp.

Searle, John R. (1982), „Eine Taxonomie illokutionärer Akte“, in: Searle, John R. (1982), Ausdruck und Bedeutung, Frankfurt/M.: Suhrkamp, pp. 17-50.

Searle, John R., Daniel Vanderveken (1985), Foundations of Illocutionary Logic, Cambridge: Cambridge University Press.

Spenader, Jennifer (2004), „Modality Realization as Contrast in Discourse“, Journal of Semantics, 21/2 (2004), pp. 113-131.

Stechow, Arnim von, Wolfgang Sternefeld (1988), Bausteine syntaktischen Wissens. Ein Lehrbuch der generativen Grammatik, Opladen: Westdeutscher Verlag.

Thrane, Torben (2004), „Hvorfor er sproget så svært at forstå når det er så let at forstå?“, in: Engerer, Volkmar (red.) (2004), Form og betydning. Bidrag til syntaks-semantik-interface i sprog og grammatik, Århus: SB-Tryk, pp. 37-70 (= Tidsskrift for Sprogforskning, 2/2 2004).

Vanderveken, Daniel (1990/1991), Meaning and speech acts, 2 vols., Cambridge: Cambridge University Press.

von Kutschera, Franz (1975), Sprachphilosophie, München: Fink.

Zaefferer, Dietmar (1979), „Sprechakttypen in einer Montague-Grammatik. Ein modelltheoretischer Ansatz zur Behandlung illokutionärer Rollen“, in: Grewendorf, Günther (Hg.) (1979), Sprechakttheorie und Semantik, Frankfurt/M.: Suhrkamp, pp. 386-417. 
Zaefferer, Dietmar (2001), „60. Modale Kategorien“, in: Haspelmath, Martin et al. (eds.) (2001), Language typology and language universals. An international handbook, Vol. 1, Berlin, New York: de Gruyter, pp. 784-816.

Zifonun, Gisela, Ludger Hoffmann, Bruno Strecker (1997), Grammatik der deutschen Sprache, 3 Bde., Berlin, New York: de Gruyter.

\section{ANMERKUNGEN}

1 Es ist traditionell zwischen Modalität und Modus zu unterscheiden (vgl. Bußmann 2002: 438f., 444 sowie Palmer 1986: 21-23), Die linguistische Terminologie spiegelt diese Distinktion im Substantivbereich wider, nicht nur im Deutschen, auch im Englischen („modality“/,mood“) oder im Dänischen („modalitet“/,modus“). Allerdings stehen in keiner der genannten Sprachen entsprechend differenzierte Adjektivableitungen zu Verfügung, die Wortform „modal“ (in allen drei Sprachen dieselbe) kann sich sowohl auf "Modalität“ als auch „Modus“ beziehen, ist also terminologisch zweideutig. Ich hoffe, dass der Kontext sowie die Mitarbeit des Lesers die schlimmsten Missverständnisse verhindern.

2 Es scheint mir ohne Zweifel, dass mit der Emergenz von Modalität in natürlichen Sprachen auch ein Sprung in der kognitiven Entwicklung des menschlichen Gehirns einhergegangen sein muss. Diesen Punkt kann ich hier nicht weiter verfolgen.

3 "The essence of modality consists in the relativization of the validity of a proposition to a set of possible worlds." (Kiefer 1998: 18)

4 Ich werde hier nicht systematisch zwischen subjektiver und objektiver epistemischer Modalität unterscheiden (vgl. Lyons 1977: 797 sowie Kiefer 1998: 7f.).

5 Ich übernehme hier die Terminologie von Cann (1993: 270ff.). Lyons (1977: 787ff.) verwendet "synthetisch“ (alle kontingenten Propositionen, egal ob wahr oder falsch), „analytisch“ (nicht-kontingent, wahr, auch „tautologisch“) sowie „kontradiktorisch“ (nicht-kontingent, falsch). Die Terminologie von synthetischen und analytischen Sätzen (Urteilen) ist v.a. in der Sprachphilosophie gängig - und ist dort von Quine auch angegriffen worden (vgl. von Kutschera 1975: 104ff., Kempson 1977: 30ff.). Die hier verwendete Terminologie ist der formalen Logik verhaftet.

6 Eine Proposition ist in der alethischen Logik notwendig wahr, wenn die Wahrheit alleine durch die Bedeutung des sie ausdrückenden Satzes garantiert wird; Erfahrung oder Beobachtung rückt also nicht an notwendig wahren Sätzen. Vgl. Kiefer (1998: 2).

7 Eine sehr kurzgefasste, aber sehr informative Darstellung modallogischer Techniken ist Davies (1994). Hier finden sich die wichtigsten Theoreme der propositionalen Modallogik, die Grundzüge einer modelltheoretischen Semantik für modale Systeme sowie Modallogik mit Quantifikatoren. Siehe auch Kiefer (1998: 2ff.) für eine informative Darstellung des Modalitätskonzeptes in der Logik. Allgemeine Einführungen in die formale Semantik sind, neben Chierchia/McConnell-Ginet (1990) und Cann (1993), Dowty/Wall/Peters (1981) sowie Lohnstein (1996).

8 Auch eine der wohl besten Einführungen in die formale Semantik, Chierchia/McConnell-Ginet (1990), referieren in ihren Kapitel zu den Modalen ausführlich Kratzers Theorie (Chierchia/McConnell-Ginet 1990: 234-241).

9 Der Konjunktiv im Deutschen ist eine sehr gut bearbeitete verbale Kategorie, insbesondere was seine Formsystematik angeht. Vgl. die entsprechenden Kapitel in den Grammatiken des Deutschen, z.B. Eisenberg (1999), Engel (1988), Heidolph/Flämig/Motsch (1981) und Zifonun/Hoffmann/Strecker (1997).

10 Man könnte auch, in Anlehnung an Kiefer (1994: 2517 sowie 1998: 9f.), Umstandsmodalität in zwei Kategorien aufspalten, „reine Umstandsmodalität“ und dispositionelle Modalität (die boulomäische vernachlässige ich). Während reine 
Umstandsmodalität als Kerngruppe Hintergründe umfasst, die als mehr oder weniger natürlich gegeben und unabhängig von einem willensbegabten Individuum gelten (In the mountains pitched roofs must be built - „In den Bergen sind die Umstände so, dass ...", vgl. Kiefer 1994: 2517), ist die dispositionelle Modalität immer auf die Möglichkeiten/Notwendigkeiten in Bezug auf ein Agens hingewiesen (Jane cannot sing today - „Janes Dispositionen sind heute so, dass ...", vgl. Kiefer 1994: 2517). Beide sind, wie Kiefer (1994: 2517) hervorhebt, mit dem Begriff der Fähigkeit verbunden. Obwohl mir eine Trennung der beiden Modalitäten danach, wo die Hintergrundeigenschaften platziert sind, möglich erscheint (ist das vom syntaktischen Subjekt denotierte Individuum agentisch und belebt, wird die dispositionelle Lesart bevorzugt, andernfalls die umstandsmodale), möchte ich Kratzers Darstellung hier folgen, um die folgende Diskussion nicht unnötig zu erschweren.

11 Kiefer argumentiert, dass die Haupttrennlinie zwischen der epistemischen Modalität und allen anderen Typen verläuft (Kiefer 1998: 10), was meinen Punkt in Verbindung mit der Sonderstellung der epistemischen Modalität auch unterstützen würde. Kiefer bemerkt z.B., dass alle Arten von Umstandsmodalitäten sowie deontische Modalität der wichtigen Implikation $\square \mathrm{p} \rightarrow \mathrm{p}$ nicht genügen, der Schluss ist nur in epistemischer Interpretation gültig (Kiefer 1998: 10). Ob die logische Eigenschaft der nicht-Validität von $\square \mathrm{p} \rightarrow \mathrm{p}$ in nicht-epistemischen Modalitäten mit der kausalen Interpretation von Umstandsmodalität zu tun hat, bleibt nachzuprüfen.

12 Mehr syntaktische Eigenschaften von modalen Ausdrücken, hierunter die komplizierten Skopusverhältnisse bei deutschen Modalverben und Kontroll- und Hebungsstrukturen epistemischer und nicht-epistemischer Modale, sind ganz kurz dargestellt in Kiefer (1998: 20-23). Allgemein zur Syntax von Kontroll- und Hebungsverben vgl. Borsley (1997) sowie Stechow/Sternefeld (1988).

13 Ich bin mir klar darüber, dass die semantischen Interpretationen, die Kratzer (1991) für die englischen Modalverben must und can anbietet, wohl nicht kommentarlos auf die deutschen Gegenstücke müssen und können übertragen werden können. Ich denke aber trotzdem, dieses Vorgehen ist vertretbar, insbesondere, weil die hier in Frage stehenden modalen Ausdrücke in beiden Sprachen in gleicher Weise an die modalen Grunddimensionen Notwendigkeit und Möglichkeit gebunden sind.

14 Obwohl die hier behandelte Idee Ballys einer durchgehenden Zweiteilung von Sätzen in einen modalen Teil und etwas, das umgangssprachlich als „Kernbedeutung“ des Satzes charakterisiert werden kann, wie sich weiter unten zeigt, Probleme mit sich bringt (z.B. umfasst der Begriff „Einstellungsprädikat“ gleichzeitig zu viel - es ist gut, dass beispielsweise ist nicht modal im eigentlichen Sinne, vgl. Kiefer 1998: 18f. - und gleichzeitig zu wenig, vgl. objektiv epistemische Modalität, die weiter unten näher behandelt wird), ist die Abtrennung der modalen Satzkomponente vom Gesamtsatz doch ein Verfahren, das in vielen Schattierungen in allen Bereichen der Modalitätsforschung wiederkehrt. In Übereinstimmung mit der linguistisch-logischen Tradition bezeichne ich hier jenen „unmodalen Satzrest“ als Proposition, wobei ich mir der ungenauen Redeweise aus der Sicht der formalen Semantik durchaus bewusst bin. Die Trennung in Modus („modale Kategorie“) und Proposition ist in neuester Zeit wieder fruchtbar gemacht worden, z.B. in den Arbeiten von Zaefferer („Eine modale Kategorie ist eine Kategorie der funktionalen Einordnung einer Proposition in den übergeordneten Zusammenhang“, Zaefferer 2001: 785).

15 Eine Parallele zu Searles Taxonomie der Sprechakte (vgl. Searle 1982), liegt nahe, obwohl Kiefer nicht explizit darauf Bezug nimmt. Für das hier folgende wäre es auch interessant $\mathrm{zu}$ sehen, inwieweit formalisierte Ansätze der Sprechakttheorie (Searle/Vanderveken 1985 sowie Vanderveken 1990/1991) eine explizite Inbezugsetzung von Satztypen, Modus und illokutionären Kräften ermöglichen (ein erster, früher Versuch ist Zaefferer 1979). Zu den relativ seltenen systematischen Anwendungen der Sprechakttheorie auf das Deutsche vgl. Hindelang (1994) sowie Rolf (1997).

16 Eine längere Diskussion des Verhältnisses zwischen Semantik und Pragmatik findet sich in Levinson (1990). 


\section{VOLKMAR ENGERER}

17 Unter Berufung auf Freges Unterscheidung von Gedanke, Urteil und Behauptung („assertion“) weitet Kiefer (1994: 2516) den sprecherorientierten Modalitätsbegriff auf Deklarative aus, wie es auch in den Paraphrasen schon zum Ausdruck kam.

18 In einem neueren Artikel wendet sich Kiefer gegen eine pauschale sprechakttheoretische Analyse deontischer Notwendigkeit und Möglichkeit, indem er die Behauptung deontischer Notwendigkeit und Möglichkeit vom Auffordern (jemandem eine Verpflichtung auferlegen) oder dem Erteilen einer Erlaubnis trennt (vgl. Kiefer 1998: 9). Ersteres sei ein rein semantisches Phänomen, die letzten beiden seien in der Sprechakttheorie zu beschreiben. Ich denke, meine Analyse kann auch dieser (einleuchtenden) Unterscheidung Rechnung tragen.

19 Kiefer, der ja das Einstellungskonzept von Modalität vertritt, stellt selbst Lyons' (1977: 798) Unterscheidung von subjektiver und objektiver Modalität relativ ausführlich dar (vgl. Kiefer 1994: 2517). Die Anerkennung objektiver Modalität („, [...] refers to reality, [...] is part of the description of the world“, Kiefer 1994: 2517) führt ihn allerdings nicht zum Schluss, dass der Begriff von Einstellung als modale Kerneigenschaft des Sprechers, hinterfragt werden müsste.

20 In einer späteren Arbeit distanziert sich Kiefer auch von dieser verallgemeinernden Fassung von Modalität und Modus: „According to this view, then, all cognitive, emotive, volitional modifications of a propositional content count as expressions of modality. This view of modality is certainly too broad and has to be narrowed down appropriately [...]“ (Kiefer 1998: 14). Im Lichte des Modalität definierenden Kriteriums als die Relativierung der Gültigkeit einer Proposition auf eine Menge möglicher Welten schließt Kiefer (1998: 18f.) eine Reihe von „Einstellungsprädikaten“ aus, hierunter eine Reihe kognitiver, alle emotive und faktive Prädikate sowie Negation.

21 Diese "Doppelmodalität” ist erkannt worden, vgl. Kiefer (1998: 17): „[...] in German modality is partly based on necessity and possibility, and partly on evidentiality." 